\title{
A Study of Birth Order and Adjustment among College Students
}

\author{
Ramesh D. Waghmare ${ }^{1 *}$
}

\section{ABSTRACT}

In the present study the birth order and Adjustment among college students has been studied. Sample of the study has been chosen from medical and engineering college students of Jalna and Aurangabad District in Maharashtra. In each 30 first born students (15 Boys and 15 Girls students). 30 second born (15 Boys and 15 Girls students) and third born students (15 Boys and 15 Girls students) the scale was used for data collection Bell's Adjustment Inventory by Lilit Sharma. 3x2 factorial design was used and data were Analysis by mean, SD and ANOVA. It has been found that there is no significance difference between first born, second born and third born students on Home, Social, Emotional and Health Adjustment.

Keywords: Birth order, Adjustment, College students

In everyday life frequent use is made of the word adjustment connotes happiness and freedom from personal problems. To other's it means an unhappy conformity to group demands and expectations. Adjustment is a process by which a living organism main taints a balance between its needs and the circumstances that influence the satisfaction of these needs and which a person varies his behavior to produce a more harmonious relationship between himself and his environment. The concept encompasses so many aspects of human adjustment that it is virtually useless in a scientific sense on the other hand the very lack of explicitness of the concept allows for the ordering and interpretation of a great variety of human active behavior along a single dimension or continuum.

Adjustment consol happiness and freedom from people problems to others it means an unhappy conformity to group demands and expectations psychologists too think way is most meaningful. The concept of adjustment means adaption to physical environment as well as to social demands no human being can live apart from his physical environment. There is action and reaction chain going on between the individual and his environment.

\footnotetext{
${ }^{1}$ Ph.D. Research Scholar, Department of Psychology, Dr. Babasaheb Ambedkar Marathwada University, Aurangabad. Maharashtra, India

*Responding Author

(C) 2016 I R Waghmare; licensee IJIP. This is an Open Access Research distributed under the terms of the Creative Commons Attribution License (http://creativecommons.org/licenses/by/2.0), which permits unrestricted use, distribution, and reproduction in any Medium, provided the original work is properly cited.
} 
The process of adjustment becomes still more complicated when his interaction with one situation comes into conflict with the requirement of other situation one situation may give rise to pleasure while the other may give rise to pain. Adjustment is somewhat more environmental while mental health is more personal in connection we can speak sensibly of a person who is well adjustment is one area of life but not in another.

\section{Birth order:}

Birth order refers to the order a child is born for example first born. Second born etc. Birth order is often believed to have a profound and lasting effect on psychological development. This assertion has at best only limited empirical support and has been repeatedly challenged by researchers; let birth order continues to have a strong presence in pop psychology and popular culture. The study of the past researcher shows noteworthy facts, Avdhesh's Jha alet al.(2012) suggests that the adjustment of the adolescents of Ahmedabad district bearing first born and second born is the same adjustment.

\section{OBJECTIVES}

To examine the home, Social, Emotional and Health adjustment among first, second and third born college students.

\section{Hypotheses}

1) There is no significant difference between first born, second born and third born on dimension home adjustment.

2) There is no significant difference between first born, second born and third born on dimension social adjustment.

3) There is no significant difference between first born, second born and third born on dimension Emotional adjustment.

4) There is no significantly difference between first born, second born and third born on dimension health adjustment.

\section{METHODOLOGY}

\section{Sample:}

The present study sample go was selected from medical and engineering college students of Jalna and Aurangabad district in Maharashtra. Total sample of the present study comprised go student. The subject selected in this sample will be used in the age group of 18 years to 22 years. Thus total sample includes as shown in the following table.

The sample designs will be used for the present study.

\section{Birth order}

\begin{tabular}{|l|l|l|l|}
\hline Gender & First born & Second born & Third born \\
\hline Male & 15 & 15 & 15 \\
\hline Female & 15 & 15 & 15 \\
\hline Total & 30 & 30 & 30 \\
\hline
\end{tabular}




\section{Variables}

Independent variables:-

1) Birth order
i) First Born
ii) Second Born
iii) Third Born

2) Gender
i) Male
ii) Female

Dependent variables:-
i) Home
ii) Social
iii) Emotional
iv) Health

Research Design

The $3 \times 2$ factorial designs will be used for the present study.

\begin{tabular}{|l|l|l|l|}
\hline & A1 & A2 & A3 \\
\hline B1 & 15 & 15 & 15 \\
\hline B2 & 15 & 15 & 15 \\
\hline
\end{tabular}
A- Birth order.
A1- First born.
A2- second born.
A3- Third born.
B- Gender
B1- Male
B2- Female.

\section{Research Tool}

\section{Bell's Adjustment Inventory}

Bell's adjustment was developed by Lalit Sharma. This inventory consists of 80 items and each item having two alternatives i.e. yes or no, subject has to give their response by choosing one from yea or no alternative. This inventory reliability was split half (odd even method) 0.897 and test retest method was 0.927 and validity Co-efficient was found to be very high 0.834 . The scoring of this inventory is very simple which can be scored simply by counting the number of correct answers marked in each area of adjustment. The sum of scores in difference area provides measure of total adjustment. High scores indicate poor adjustment and low scores indicate better adjustment.

\section{Research Procedure}

The following research methodology will be used in the present study. The primary information will be gathered by giving personal information form to each student. The students were called in small group 05 to 10 students. To fill the inventories subjects were given general instructions belongs. The students were provided the Bell's adjustment Inventory. Data were obtained by using particular scoring palter standardized for each test. 


\section{A Study of Birth Order and Adjustment among College Students}

\section{Statistical Analysis and Discussion}

The mean and SD with graphical representation for Birth order on students adjustment was analyzed. A 3x2 factorial design was used and subjected to adequate o statistical analysis of variance (ANOVA) in order t examine the roll of main variables and to study their main as well as subsequently on students adjustment.

Table No.01, Show the mean, SD and ' $F$ ' Value of Home Adjustment

\begin{tabular}{|l|l|l|l|l|l|l|}
\hline Birth Order & Mean & SD & N & df & F Value & Significance \\
\hline First born & 6.03 & 2.69 & 30 & & \multirow{2}{*}{0.632} & \multirow{2}{*}{ NS } \\
\hline Second born & 5.33 & 2.49 & 30 & 87 & & \\
\hline Third born & 5.50 & 2.38 & 30 & & & \\
\hline
\end{tabular}

(Table value at 0.05 level -4.01 and 0.01 level -2.71)

From the table No. 01 it is observed that the mean score of the first born college students is 6.03 and SD 2.69, followed by second born college students whose mean score is 5.33 and SD 2.49 and third born college students mean score is 5.50 and SD 2.38, but on the basis of mean observation it would that mean difference 0.7 among first and second bond, 0.53 among first and third born and mean difference 0.17 among second and third born. The F value 0.632 is not significant at both levels, at a glance those first born students shows miner high score than second and third born students. In the present study was "there is no significance difference between first born, second born and third born students with dimension on home adjustment. Birth order effect represent the home adjustment was not significant (F- 0.632, 3 and 87, P> 0.01 and 0.05 ). This is no significant 0.01 and 0.05 levels because they obtained ' $F$ ' value are low than table value at 0.05 and 0.01 levels. In this indicate that first, second and third born college students not differ from home adjustment. The finding of the supported the first hypothesis, they are first hypothesis accepted the present study. It indicates that birth order does not influence significantly the home adjustment and home environment does not make significant difference in the person.

Table No.02, Show the mean, SD and ' $F$ ' Value of Social Adjustment

\begin{tabular}{|l|l|l|l|l|l|l|}
\hline Birth Order & Mean & SD & N & df & F Value & Significance \\
\hline First born & 6.53 & 1.54 & 30 & & \multirow{2}{*}{ N } & \\
\cline { 1 - 4 } Second born & 6.56 & 2.34 & 30 & & \\
\cline { 1 - 4 } Third born & 6.30 & 1.82 & 30 & & \\
\hline
\end{tabular}

(Table value at 0.05 level -4.01 and 0.01 level -2.71)

From the table No. 02 it is observed that the mean score of the first born college students is 6.53 and SD 1.54, followed by second born college students whose mean score is 6.56 and SD 2.34 and third born college students mean score is 6.30 and SD 1.82, but on the basis of mean observation it would that mean difference 0.03 among first and second bond, 0.23 among first and third born and mean difference 0.26 among second and third born. The F value 0.17 is not 


\section{A Study of Birth Order and Adjustment among College Students}

significant at both levels, at a glance those second born students shows miner high score than first and third born students. In the present study was "there is no significance difference between first born, second born and third born students with dimension on Social adjustment. Birth order effect represent the Social adjustment was not significant (F- 0.17, 3 and 87, P> 0.01 and 0.05 ). This is no significant 0.01 and 0.05 levels because they obtained ' $F$ ' value are low than table value at 0.05 and 0.01 levels. In this indicate that first, second and third born college students not differ from home adjustment. The finding of the supported the second hypothesis, they are second hypothesis accepted the present study. It indicates that birth order does not influence significantly the Social adjustment and Social environment does not make significant difference in the person.

Table No.03, Show the mean, SD and 'F' Value of Emotional Adjustment

\begin{tabular}{|l|l|l|l|l|l|l|}
\hline Birth Order & Mean & SD & N & df & F Value & Significance \\
\hline First born & 9.66 & 2.82 & 30 & & \multirow{2}{*}{$*$} & \multirow{2}{*}{ NS } \\
\cline { 1 - 3 } Second born & 9.06 & 1.92 & 30 & & 0.94 & \\
\hline Third born & 9.90 & 2.45 & 30 & & & \\
\hline
\end{tabular}

(Table value at 0.05 level -4.01 and 0.01 level -2.71)

From the table No. 03 it is observed that the mean score of the first born college students is 9.66 and SD 2.82, followed by second born college students whose mean score is 9.06 and SD 1.92 and third born college students mean score is 9.90 and SD 2.45, but on the basis of mean observation it would that mean difference 0.6 among first and second bond, 0.24 among first and third born and mean difference 0.84 among second and third born. The F value 0.94 is not significant at both levels, at a glance those third born students shows miner high score than second and first born students. In the present study was "there is no significance difference between first born, second born and third born students with dimension on Emotional adjustment. Birth order effect represent the Emotional adjustment was not significant (F- 0.94, 3 and $87, \mathrm{P}>0.01$ and 0.05 ). This is no significant 0.01 and 0.05 levels because they obtained ' $\mathrm{F}$ ' value are low than table value at 0.05 and 0.01 levels. In this indicate that first, second and third born college students not differ from Emotional adjustment. The finding of the supported the third hypothesis, they are third hypothesis accepted the present study. It indicates that birth order does not influence significantly the Emotional adjustment and Emotional environment does not make significant difference in the person.

Table No.04, Show the mean, SD and ' $F$ ' Value of Health Adjustment

\begin{tabular}{|l|l|l|l|l|l|l|}
\hline Birth Order & Mean & SD & N & df & F Value & Significance \\
\hline First born & 5.20 & 2.07 & 30 & \multirow{2}{*}{$*$} & \multirow{2}{*}{ NS } \\
\cline { 1 - 4 } Second born & 4.50 & 2.02 & 30 & & & \\
\hline Third born & 5.20 & 2.49 & 30 & & \\
\hline
\end{tabular}

(Table value at 0.05 level -4.01 and 0.01 level -2.71 ) 


\section{A Study of Birth Order and Adjustment among College Students}

From the table No. 04 and Graph No. 04 it is observed that the mean score of the first born college students is 5.20 and SD 2.07, followed by second born college students whose mean score is 4.50 and SD 2.02 and third born college students mean score is 5.20 and SD 2.49, but on the basis of mean observation it would that mean difference 0.30 among first and second bond, 0.00 among first and third born and mean difference 0.30 among second and third born. The $\mathrm{F}$ value 1.00 is not significant at both levels, at a glance those second born students shows miner high score than first and third born students. In the present study was "there is no significance difference between first born, second born and third born students with dimension on health adjustment. Birth order effect represent the health adjustment was not significant (F-1.00, 3 and 87, P> 0.01 and 0.05 ). This is no significant 0.01 and 0.05 levels because they obtained ' $F$ ' value are low than table value at 0.05 and 0.01 levels. In this indicate that first, second and third born college students not differ from Health adjustment. The finding of the supported the fourth hypothesis, they are fourth hypothesis accepted the present study. It indicates that birth order does not influence significantly the Health adjustment and Health environment does not make significant difference in the person.

\section{CONCLUSION}

There no significance difference between first born, second born and third born on Home Adjustment, Social Adjustment, Emotional Adjustment and Health Adjustment.

\section{THE LIMITATION OF THE STUDY}

1) The findings of the study are based on very small sample.

2) The sample was selected only Jalna District in Maharashtra.

3) The study was use only medical and engineering college students.

4) The study was selected students are only 18 to 21 years.

\section{REFERENCES}

Adms, H.E.,(1972) Psychology of Adjustment, New York; Ronald prels.

Baker, R.W. and Siryk, B., (1984) Measuring Adjustment to college. Journal of counseling psychology, 31,179-189.

Bell, H.V., (1934) Manual for the Adjustment Inventory (students form) standard, californa; Stanford university press.

Calhoun, J.F. et.all. (1978) Adjustment: A way of Handling problem random house, psychology of Adjustment and Human relations, 16-28.

Davis, J.N.,(1997) Birth order , Sibship size and status in modern Canada, Human Nature, 8; 205-230.

Garred, H. E.,(1967) Statistics in psychology and Education (4 ${ }^{\text {th }}$ ed.) Bombay vakils, Feffer and simons pvt.Ltd.

Jha, A. S., Dwlvedi, A, and singh, P. V., (2012) Effect of Birth order, Gender and medium of Instruction of the adolescents on their adjustment. Voice of Research, 1 (1), 25-26. 
Jocobs, B. S. and Moss, H. A., (1967) Birth order and sex of siblingas determinants of mother Infant Interaction, child Development, 47, 315-322.

Salmon, C. A. and Daly, D. (1998) The Impact of Birth order on familial sentiment; middle borns are Different, Human Behavior and Evolution, 19;2, 99-212.

Shaffer, L.F. (1970) The psychology of Adjustment, New York; Houghton Mifilin.

Sulloway, F. J. (1995) Birth order and Evolutionary psychology; A meta Analytic overview. 